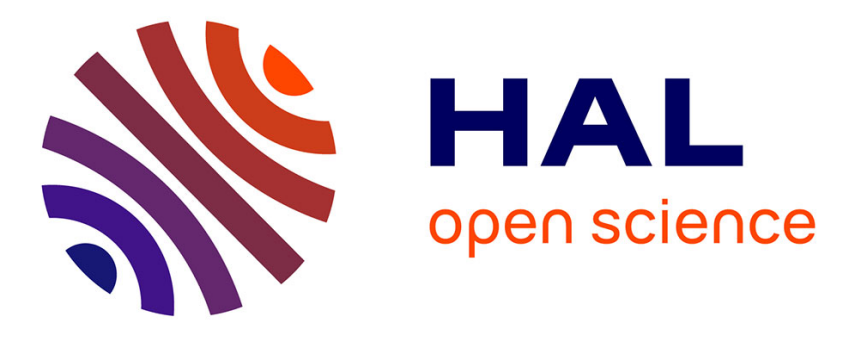

\title{
Output Feedback Control of a Class of Stochastic Hybrid Systems
}

Samir Aberkane, Jean-Christophe Ponsart, Mickael Rodrigues, Dominique

Sauter

\section{- To cite this version:}

Samir Aberkane, Jean-Christophe Ponsart, Mickael Rodrigues, Dominique Sauter. Output Feedback Control of a Class of Stochastic Hybrid Systems. Automatica, 2008, 44 (5), pp.1325-1332. 10.1016/j.automatica.2007.09.021 . hal-00201094

\section{HAL Id: hal-00201094 \\ https://hal.science/hal-00201094}

Submitted on 23 Dec 2007

HAL is a multi-disciplinary open access archive for the deposit and dissemination of scientific research documents, whether they are published or not. The documents may come from teaching and research institutions in France or abroad, or from public or private research centers.
L'archive ouverte pluridisciplinaire HAL, est destinée au dépôt et à la diffusion de documents scientifiques de niveau recherche, publiés ou non, émanant des établissements d'enseignement et de recherche français ou étrangers, des laboratoires publics ou privés. 


\title{
Output Feedback Control of a Class of Stochastic Hybrid Systems *
}

\author{
Samir Aberkane ${ }^{\text {a }}$, Jean Christophe Ponsart ${ }^{a}$,Mickael Rodrigues ${ }^{\text {a }}$,Dominique Sauter ${ }^{\text {a }}$ \\ ${ }^{\text {a }}$ CRAN - CNRS UMR 7039, Université Henri Poincaré, BP 239, F-54506 Vandœuvre-lès-Nancy Cedex
}

\begin{abstract}
This paper deals with static output feedback control of a class of reconfigurable systems with Markovian Parameters and state-dependent noise. The main contribution is to formulate conditions for multi-performance design related to this class of stochastic hybrid systems. The specifications and objectives under consideration include stochastic stability, $\mathcal{H}_{2}$ and $\mathcal{H}_{\infty}$ performances. Another problematic related to a more general class of stochastic hybrid systems, known as Markovian Jump Linear Systems (MJLS), is also addressed. This problematic concerns the mode-independent output feedback control of MJLS. The obtained results are illustrated on a numerical example.
\end{abstract}

Key words: Reconfigurable Control; Markovian Jumping Parameters; $\mathcal{H}_{2} / \mathcal{H}_{\infty}$ Control; Static Output Feedback.

\section{Introduction}

Fault tolerant control systems (FTCS) aim at guaranteeing that a process keeps fulfilling its mission even in the presence of failures, although possibly in a degraded mode. For the design problem, two basic approaches have been studied: passive fault-tolerance rely on robust control principles where the faults are considered as modeling uncertainties. Active fault tolerant control (AFTC), on the other hand, involves automatically detecting and identifying the failed components using a fault detection and identification (FDI) scheme and then reconfiguring the control law on-line in response to the FDI algorithm decisions. Despite the evident interaction between FDI and reconfiguration algorithms, the research on FDI and reconfiguration methods has often evolved separately. Typically, in the reconfiguration literature, it is usually assumed that a perfect FDI device is available (i.e. no detection delays, no false alarms, no missed detection ...etc.). On the other hand, an FDI algorithm is judged satisfactory if it detects failures quickly and generates few false alarms, regardless of the performance of the closed loop reconfigured system. The first purpose of this paper is then to use a mathematical model that includes in the same analysis framework

\footnotetext{
ॠ This paper was not presented at any IFAC meeting. Corresponding author S. Aberkane. Tel. +33 3836844 80. Fax +33383684462 .
}

Email address: samir.aberkane@cran.uhp-nancy.fr (Samir Aberkane). the FDI and reconfiguration algorithms. Such a model was initially proposed by $[18,19]$. The author shows that when the FDI output is feedback into a reconfiguration closed-loop control, a short detection delay and a low false alarm rate do not guarantee stable operation of the reconfigurable system. This makes the synthesis of reconfigurable controllers that take explicitly into account the inherent imperfections of the FDI process of great importance.

The proposed model in [18] belongs to the class of MJLS. In MJLS, a single jump process is used to describe the random variations affecting the system parameters. This process is represented by a finite state Markov chain and is called the plant regime mode. The theory of stability, optimal control and $\mathcal{H}_{2} / \mathcal{H}_{\infty}$ control, as well as important applications of such systems, can be found in several papers in the current literature, for instance in $[3,4,2,6,8,9,13,14,19]$. In the class of stochastic hybrid systems introduced in $[18,19]$, two random processes are defined: the first random process represents system components failures and the second random process represents the FDI process used to reconfigure the control law. The problem of stochastic stability of active fault tolerant control systems with Markovian parameters (AFTC$\mathrm{SMP}$ ) in the presence of noise, parameter uncertainties, detection errors, detection delays and actuator saturation limits as well as $\mathcal{H}_{\infty}$ performance analysis have been investigated in $[1,16,17,21,22]$.

On the other hand, one of the most challenging open problems in control theory is the synthesis of fixed-order or static output feedback controllers that meet desired 
performances and specifications [25]. Among all variations of this problem, this note is concerned with the problem of static output feedback control of continuous time AFTCSMP with state-dependent noise. The main contribution is to formulate conditions for multiperformance design related to this class of stochastic hybrid systems. The specifications and objectives under consideration include stochastic stability and $\mathcal{H}_{2} / \mathcal{H}_{\infty}$ performances. Another problematic related to a more general class of stochastic hybrid systems, (MJLS), is also addressed. This problematic concerns the modeindependent static output feedback control of this class of systems. Most of the developed results in the field of MJLS control are obtained under the restrictive assumption of control with complete state observation (Markov and system states). However, the access to the system mode may not be possible in some circumstances, which limits the use of such controllers. For instance, if the changes in the Markov chain are associated to failures of components of non-critical significance, or more generally if some changes are difficult to measure, it is quite possible that the associated Markov states are not accessible to controller. This scenario impose serious limitations in the analytical front [10]. One can distinguish two approaches for the design of mode-independent controllers. The first one uses a constant Lyapunov function $[5,7,11]$, which leads to a linear matrix inequality (LMI) formulation of the design problem. The second one uses mode-dependent Lyapunov function and therefore leads to less conservative results. This approach usually leads to bilinear matrix inequality (BMI) problem. All the aforementioned works are based on the critical assumption of the complete access to the state vector. Practically, this assumption may sometimes be hard to satisfy and therefore, the developed results are restrictive. To the best of our knowledge, to date the problem of static output-feedback $\mathcal{H}_{2} / \mathcal{H}_{\infty}$ control of continuoustime MJLS with a non-accessible jumping parameter has not yet been fully addressed. Our approach belongs to the class of methods that use mode-dependent Lyapunov functions.

This paper is organized as follows: Section 2 describes the dynamical model of the system. A brief summary of basic stochastic terms and results are given in Section 3. Section 4 addresses the stochastic stabilization problematic. Section 5 considers the $\mathcal{H}_{2} / \mathcal{H}_{\infty}$ control problem. In Section 6, a numerical algorithm is provided and its running is illustrated on a numerical example. Section 7 concludes this paper.

Notations. $\mathbb{R}^{m \times n}$ is the set of $m$-by- $n$ real matrices and $\mathbb{S}^{n}$ is the subset of symmetric matrices in $\mathbb{R}^{n \times n}$. $A^{\prime}$ is the transpose of the matrix $A$. The notation $X \geq Y$ ( $X>Y$, respectively), where $X$ and $Y$ are symmetric matrices, means that $X-Y$ is positive semi-definite (positive definite, respectively); $\mathbb{I}$ and $\mathbf{0}$ are identity and zero matrices of appropriate dimensions, respectively; $\mathcal{E}\{\cdot\}$ denotes the expectation operator with respect to some probability measure $P ; L^{2}[0, \infty)$ stands for the space of square-integrable vector functions over the in- terval $[0, \infty) ;\|\cdot\|$ refers to either the Euclidean vector norm or the matrix norm, which is the operator norm induced by the standard vector norm; $\|\cdot\|_{2}$ stands for the norm in $L^{2}[0, \infty)$; while $\|\cdot\|_{\mathcal{E}_{2}}$ denotes the norm in $L^{2}((\Omega, \mathcal{F}, P),[0, \infty)) ;(\Omega, \mathcal{F}, P)$ is a probability space. We also denote $\bullet(t)=\bullet_{t}$, and the initial conditions $\bullet\left(t_{0}\right)=\bullet_{0}$. In block matrices, $\star$ indicates symmetric terms: $\left[\begin{array}{cc}A & B \\ B^{\prime} & C\end{array}\right]=\left[\begin{array}{cc}A & \star \\ B^{\prime} & C\end{array}\right]=\left[\begin{array}{cc}A & B \\ \star & C\end{array}\right]$.

\section{Dynamical model}

To describe the class of linear systems with Markovian jumping parameters that we deal with in this paper, let us fix a complete probability space $(\Omega, \mathcal{F}, P)$. The dynamical model of the reconfigurable system with Markovian process and state dependent noise, defined in the fundamental probability space $(\Omega, \mathcal{F}, P)$, is described by the following differential equations:

$$
\varphi:\left\{\begin{aligned}
d x_{t}=A\left(\xi_{t}\right) x_{t} d t+B\left(\eta_{t}\right) u_{t} d t+E\left(\xi_{t}, \eta_{t}\right) w_{t} d t & \\
& +\sum_{l=1}^{v} \mathbb{W}_{l}\left(\xi_{t}, \eta_{t}\right) x_{t} d \varpi_{l t} \\
y_{t}= & C_{2} x_{t}+D_{2}\left(\xi_{t}, \eta_{t}\right) w_{t} \\
z_{t}= & C_{1} x_{t}+D_{1}\left(\eta_{t}\right) u\left(y_{t}, \psi_{t}, t\right)
\end{aligned}\right.
$$

where $x_{t} \in \mathbb{R}^{n}$ is the system state, $u_{t} \in \mathbb{R}^{r}$ is the system input, $y_{t} \in \mathbb{R}^{q}$ is the system measured output, $z_{t} \in \mathbb{R}^{p}$ is the controlled output, $w_{t} \in \mathbb{R}^{m}$ is the system external disturbance, $\xi_{t}, \eta_{t}$ and $\psi_{t}$ represent the plant component failure process, the actuator failure process and the FDI process, respectively. $\xi_{t}, \eta_{t}$ and $\psi_{t}$ are separable and measurable Markov processes with finite state spaces $Z=\{1,2, \ldots, z\}, S=\{1,2, \ldots, s\}$ and $R=\{1,2, \ldots, r\}$, and transition rate matrices $\Pi=\left[\pi_{i j}, i \in Z, j \in Z\right], \Upsilon=$ $\left[\nu_{i j}, i \in S, j \in S\right], \Lambda=\left[\lambda_{i v}^{k l}, i \in R, v \in R, k \in Z, l \in S\right]$ respectively. $\pi_{i j}$ is the plant components failure rate, and $\nu_{k l}$ is the actuator failure rate. Here, $\lambda_{i v}^{k l}$ represents the transition rate from $i$ to $v$ for the Markov process $\psi(t)$ conditioned on $\xi=k \in Z$ and $\eta=l \in S$. Depending on the values of $i, v \in R, k \in Z$ and $l \in S$, various interpretations, such as rate of false detection and isolation, rate of correct detection and isolation, false alarm recovery rate, etc, can be given to $\lambda_{i v}^{k l}[17,24] . \varpi_{t}=\left[\varpi_{1 t} \ldots \varpi_{v t}\right]^{\prime}$ is a $v$-dimensional standard Wiener process on a given probability space $(\Omega, \mathcal{F}, P)$, that is assumed to be independent of the Markov processes.

In AFTC, we consider that the control law is only a function of the mesurable FDI process $\psi(t)$. Therefore, we introduce a static output feedback compensator $\left(\varphi_{s}\right)$ of the form:

$$
\varphi_{s}:\left\{u_{t}=\mathcal{K}\left(\psi_{t}\right) y_{t}\right.
$$

Applying the controller $\varphi_{s}$ to $\varphi$, we obtain the following closed loop system:

$$
\varphi_{c l}:\left\{\begin{aligned}
d x_{t}= & \bar{A}\left(\xi_{t}, \eta_{t}, \psi_{t}\right) x_{t} d t+\bar{E}\left(\xi_{t}, \eta_{t}, \psi_{t}\right) w_{t} d t \\
& +\sum_{l=1}^{v} \mathbb{W}_{l}\left(\xi_{t}, \eta_{t}\right) x_{t} d \varpi_{l t} \\
y_{t}= & C_{2} x_{t}+D_{2}\left(\xi_{t}, \eta_{t}\right) w_{t} \\
z_{t}= & \bar{C}_{1}\left(\eta_{t}, \psi_{t}\right) x_{t}+\bar{D}_{1}\left(\xi_{t}, \eta_{t}, \psi_{t}\right) w_{t}
\end{aligned}\right.
$$


where

$$
\begin{aligned}
& {\left[\begin{array}{cc}
\bar{A}\left(\xi_{t}, \eta_{t}, \psi_{t}\right) & \bar{E}\left(\xi_{t}, \eta_{t}, \psi_{t}\right) \\
\bar{C}_{1}\left(\eta_{t}, \psi_{t}\right) & \bar{D}_{1}\left(\xi_{t}, \eta_{t}, \psi_{t}\right)
\end{array}\right]=} \\
& {\left[\begin{array}{cc}
A\left(\xi_{t}\right) & E\left(\xi_{t}, \eta_{t}\right) \\
C_{1} & 0
\end{array}\right]+\left[\begin{array}{c}
B\left(\eta_{t}\right) \\
D_{1}\left(\eta_{t}\right)
\end{array}\right] \mathcal{K}\left(\psi_{t}\right)\left[\begin{array}{ll}
C_{2} & D_{2}\left(\xi_{t}, \eta_{t}\right)
\end{array}\right]}
\end{aligned}
$$

For notational simplicity, we will denote $\bullet\left(\xi_{t}\right)=\bullet_{i}$ when $\xi_{t}=i \in Z, \bullet\left(\eta_{t}\right)=\bullet_{j}, \bullet\left(\xi_{t}, \eta_{t}\right)=\bullet_{i j}$ when $\xi_{t}=i \in$ $Z, \eta_{t}=j \in S$ and $\bullet\left(\psi_{t}\right)=\bullet{ }_{k}$ when $\psi_{t}=k \in R$.

\section{Definitions and Basic Results}

In this section, we will first give basic definitions related to stochastic stability notions and then we will summarize some results about stochastic stability of reconfigurable systems with Markovian processes.

\subsection{Stochastic Stability}

For system (1), when $u_{t} \equiv 0$ for all $t \geq 0$, we have the following definition.

Definition 1. System (1) is said to be internally exponentially stable in the mean square sense (IESS), if there exist positive constants $\alpha$ and $\beta$ such that the solution of

$$
d x_{t}=A\left(\xi_{t}\right) x_{t} d t+\sum_{l=1}^{v} \mathbb{W}_{l}\left(\xi_{t}, \eta_{t}\right) x_{t} d \varpi_{l t}
$$

satisfies the following inequality

$$
\mathcal{E}\left\{\left\|x_{t}\right\|^{2}\right\} \leq \beta\left\|x_{0}\right\|^{2} \exp \left[-\alpha\left(t-t_{0}\right)\right]
$$

for arbitrary initial conditions $\left(x_{0}, \xi_{0}, \eta_{0}\right)$.

Definition 2. System (1) is said to be internally exponentially stabilizable in the mean square sense by static output feedback, if there exists a control $\varphi_{s}$ such that the closed loop system (3) is IESS.

The following proposition gives a necessary and sufficient condition for IESS of the system (3).

Proposition 1. System (3) is IESS if and only if there exists symmetric positive-definite matrices $\mathcal{P}_{i j k}, i \in Z$, $j \in S$ and $k \in R$ such that:

$$
\begin{aligned}
\bar{A}_{i j k}^{\prime} \mathcal{P}_{i j k} & +\mathcal{P}_{i j k} \bar{A}_{i j k}+\sum_{l=1}^{v} \mathbb{W}_{l i j}^{\prime} \mathcal{P}_{i j k} \mathbb{W}_{l i j}+\sum_{h \in Z} \pi_{i h} \mathcal{P}_{h j k} \\
& +\sum_{l \in S} \nu_{j l} \mathcal{P}_{i l k}+\sum_{v \in R} \lambda_{k v}^{i j} \mathcal{P}_{i j v}=\beth_{i j k}<0
\end{aligned}
$$

where: $\bar{A}_{i j k}=A_{i}+B_{j} \mathcal{K}_{k} C_{2}$

Proof. Due to page limit, the proof of this proposition is not detailed here. It is based on classical tools from stochastic stability analysis [15].

Proposition 2. If system (3) is IESS, for every $w=$ $\left\{w_{t} ; t \geq 0\right\} \in L_{2}[0, \infty)$, we have that $x=\left\{x_{t} ; t \geq 0\right\} \in$ $L_{2}((\Omega, \mathcal{F}, P),[0, \infty))$, i.e., $\mathcal{E}\left\{\int_{0}^{\infty} x_{t}^{T} x_{t} d t\right\}<\infty$, for any initial conditions.

The proof of proposition 2 is omitted. It follows the same arguments as for the proof of proposition 5.1 in [1].

\subsection{Matrix Ellipsoids}

Through this paper, a particular set of matrices is used. In the literature, these sets are referred to as matrix ellipsoids of $\mathbb{R}^{(m \times p)}$.

Definition 3. [20] Given three matrices $\mathbb{X} \in \mathbb{S}^{q}, \mathbb{Y} \in$ $\mathbb{R}^{q \times r}$ and $\mathbb{Z} \in \mathbb{S}^{r}$, the $\{\mathbb{X}, \mathbb{Y}, \mathbb{Z}\}$-ellipsoid of $\mathbb{R}^{r \times q}$ is the set of matrices $\mathcal{K}$ satisfying the following matrix inequalities:

$$
\mathbb{Z}>0 ; \quad\left[\begin{array}{ll}
\mathbb{I} & \mathcal{K}^{\prime}
\end{array}\right]\left[\begin{array}{ll}
\mathbb{X} & \mathbb{Y} \\
\star & \mathbb{Z}
\end{array}\right]\left[\begin{array}{l}
\mathbb{I} \\
\mathcal{K}
\end{array}\right] \leq 0
$$

By definition, $\mathcal{K}_{0}=-\mathbb{Z}^{-1} \mathbb{Y}^{\prime}$ is the center of the ellipsoid and $R=\mathcal{K}_{0}^{\prime} \mathbb{Z}_{0}-\mathbb{X}$ is the radius. Inequality (6) can also be written as

$$
\mathbb{Z}>0 ; \quad\left(\mathcal{K}-\mathcal{K}_{0}\right)^{\prime} \mathbb{Z}\left(\mathcal{K}-\mathcal{K}_{0}\right) \leq R
$$

This definition shows that matrix ellipsoids are special cases of matrix sets defined by quadratic matrix inequality. Some properties of these sets are:

i) A matrix ellipsoid is a convex set;

ii) $\{\mathbb{X}, \mathbb{Y}, \mathbb{Z}\}$-ellipsoid is nonempty iff the radius $(R \geq 0)$ is positive semi definite. This property can also be expressed as

$$
\mathbb{X} \leq \mathbb{Y} \mathbb{Z}^{-1} \mathbb{Y}^{\prime}
$$

\section{Stochastic Stabilization}

In this section, we shall address the problem of finding all static compensators $\left(\varphi_{s}\right)$, as defined in section 2 , such that the closed loop system $\left(\varphi_{c l}\right)$ becomes IESS. To this end, we use proposition 1 and the idea of synthesizing ellipsoidal sets of controllers [20] to get the following necessary and sufficient conditions for the IESS of the system (3).

Proposition 3. System (1) is internally exponentially stabilisable in the mean square sense by static outputfeedback if and only if there exists matrices $\mathcal{P}_{i j k}=$ $\mathcal{P}_{i j k}^{\prime}>0, \mathbb{X}_{k} \in \mathbb{S}^{q}, \mathbb{Y}_{k} \in \mathbb{R}^{q \times r}$ and $\mathbb{Z}_{k}=\mathbb{Z}_{k}^{\prime}>0$ that simultaneously satisfy the following LMI constraints

$$
\begin{aligned}
{\left[\begin{array}{rr}
\mathbb{I} & \mathbf{0} \\
A_{i} & B_{j}
\end{array}\right]^{\prime} } & {\left[\begin{array}{cc}
\Theta_{i j k} & \mathcal{P}_{i j k} \\
\mathcal{P}_{i j k} & \mathbf{0}
\end{array}\right]\left[\begin{array}{cc}
\mathbb{I} & \mathbf{0} \\
A_{i} & B_{j}
\end{array}\right]<} \\
& {\left[\begin{array}{cc}
C_{2} & \mathbf{0} \\
\mathbf{0} & \mathbb{I}
\end{array}\right]^{\prime}\left[\begin{array}{cc}
\mathbb{X}_{k} & \mathbb{Y}_{k} \\
\star & \mathbb{Z}_{k}
\end{array}\right]\left[\begin{array}{cc}
C_{2} & \mathbf{0} \\
\mathbf{0} & \mathbb{I}
\end{array}\right] }
\end{aligned}
$$

and the nonlinear inequalities constraints

$$
\mathbb{X}_{k} \leq \mathbb{Y}_{k} \mathbb{Z}_{k}^{-1} \mathbb{Y}_{k}^{\prime}
$$

$\forall i \in Z, j \in S$ and $k \in R$, where

$$
\begin{aligned}
\Theta_{i j k}=\sum_{l=1}^{v} \mathbb{W}_{l i j}^{\prime} \mathcal{P}_{i j k} \mathbb{W}_{l i j}+\sum_{h \in Z} \pi_{i h} \mathcal{P}_{h j k} & +\sum_{l \in S} \nu_{j l} \mathcal{P}_{i l k} \\
& +\sum_{v \in R} \lambda_{k v}^{i j} \mathcal{P}_{i j v}
\end{aligned}
$$


Let $\left\{\mathcal{P}_{i j k}, \mathbb{X}_{k}, \mathbb{Y}_{k}, \mathbb{Z}_{k}\right\}$ be a solution, then the nonempty $\left\{\mathbb{X}_{k}, \mathbb{Y}_{k}, \mathbb{Z}_{k}\right\}$-ellipsoids are sets of stabilizing gains.

\section{Proof.}

Sufficiency. Assume that (9)-(10) are satisfied for some $\left\{\mathcal{P}_{i j k}, \mathbb{X}_{k}, \mathbb{Y}_{k}, \mathbb{Z}_{k}\right\}$ matrices. Due to the properties of matrix ellipsoids, the $\left\{\mathbb{X}_{k}, \mathbb{Y}_{k}, \mathbb{Z}_{k}\right\}$-ellipsoids are nonempty. Take any element $\mathcal{K}_{k}$. The LMI (9) implies that for all

$$
\begin{aligned}
\left(\begin{array}{ll}
x_{t}^{\prime} & u_{t}^{\prime}
\end{array}\right) \neq 0 & \left(\begin{array}{c}
x_{t} \\
A_{i} x_{t}+B_{j} u_{t}
\end{array}\right)^{\prime} \\
& {\left[\begin{array}{cc}
\Theta_{i j k} & \mathcal{P}_{i j k} \\
\mathcal{P}_{i j k} & \mathbf{0}
\end{array}\right]\left(\begin{array}{c}
x_{t} \\
A_{i} x_{t}+B_{j} u_{t}
\end{array}\right)<} \\
& \left(\begin{array}{c}
C_{2} x_{t} \\
u_{t}
\end{array}\right)^{\prime}\left[\begin{array}{cc}
\mathbb{X}_{k} & \mathbb{Y}_{k} \\
\star & \mathbb{Z}_{k}
\end{array}\right]\left(\begin{array}{c}
C_{2} x_{t} \\
u_{t}
\end{array}\right)
\end{aligned}
$$

Definition 3 implies that for all nonzero trajectories

$$
x_{t}^{\prime} \rrbracket_{i j k} x_{t}<y_{t}^{\prime}\left[\mathbb{I} \mathcal{K}_{k}^{\prime}\right]\left[\begin{array}{cc}
\mathbb{X}_{k} & \mathbb{Y}_{k} \\
\star & \mathbb{Z}_{k}
\end{array}\right]\left[\begin{array}{c}
\mathbb{I} \\
\mathcal{K}_{k}
\end{array}\right] y_{t} \leq 0
$$

Then, the closed-loop exponential stochastic stability follows from proposition 1 for the quadratic stochastic Lyapunov function $\vartheta\left(\xi_{t}, \eta_{t}, \psi_{t}\right)=x_{t}^{\prime} \mathcal{P}\left(\xi_{t}, \eta_{t}, \psi_{t}\right) x_{t}$.

Necessity. Assume $\mathcal{K}_{k}$ are stabilizing static output feedback gains and $\vartheta\left(\xi_{t}, \eta_{t}, \psi_{t}\right)=x_{t}^{\prime} \mathcal{P}\left(\xi_{t}, \eta_{t}, \psi_{t}\right) x_{t}$ is a stochastic Lyapunov function. Then from proposition 1 , we have

$$
\begin{aligned}
& {\left[\begin{array}{ll}
\mathcal{K}_{k} C_{2} & -\mathbb{I}
\end{array}\right]\left(\begin{array}{c}
x_{t} \\
u_{t}
\end{array}\right)=0 \Rightarrow} \\
& \left(\begin{array}{l}
x_{t} \\
u_{t}
\end{array}\right)^{\prime}\left[\begin{array}{cc}
\mathbb{I} & \mathbf{0} \\
A_{i} & B_{j}
\end{array}\right]^{\prime}\left[\begin{array}{cc}
\Theta_{i j k} & \mathcal{P}_{i j k} \\
\mathcal{P}_{i j k} & \mathbf{0}
\end{array}\right]\left[\begin{array}{cc}
\mathbb{I} & \mathbf{0} \\
A_{i} & B_{j}
\end{array}\right]\left(\begin{array}{l}
x_{t} \\
u_{t}
\end{array}\right)<0
\end{aligned}
$$

Based on the Finsler Lemma [23], there exist scalars $\tau_{i j k}$ such that

$$
\begin{aligned}
& {\left[\begin{array}{cc}
\mathbb{I} & \mathbf{0} \\
A_{i} & B_{j}
\end{array}\right]^{\prime}\left[\begin{array}{cc}
\Theta_{i j k} & \mathcal{P}_{i j k} \\
\mathcal{P}_{i j k} & \mathbf{0}
\end{array}\right]\left[\begin{array}{cc}
\mathbb{I} & \mathbf{0} \\
A_{i} & B_{j}
\end{array}\right]<} \\
& \tau_{i j k}\left[\begin{array}{ll}
\mathcal{K}_{k} C_{2} & -\mathbb{I}
\end{array}\right]^{\prime}\left[\begin{array}{ll}
\mathcal{K}_{k} C_{2} & -\mathbb{I}
\end{array}\right] \leq \varepsilon_{k}\left[\begin{array}{ll}
\mathcal{K}_{k} C_{2} & -\mathbb{I}
\end{array}\right]^{\prime}\left[\begin{array}{ll}
\mathcal{K}_{k} C_{2} & -\mathbb{I}
\end{array}\right]
\end{aligned}
$$

where $\varepsilon_{k}=\max _{i, j}\left(\tau_{i j k}\right)$. The inequality (9) is obtained with $\mathbb{X}_{k}=\varepsilon_{k} \mathcal{K}_{k}^{\prime} \mathcal{K}_{k}, \mathbb{Y}_{k}=-\varepsilon_{k} \mathcal{K}_{k}^{\prime}, \mathbb{Z}_{k}=\varepsilon_{k} \mathbb{I}$. (14) implies $\mathbf{0}<\mathbb{Z}_{k}$. Hence the proof is complete.

Mode-Independent Stabilization of MJLS: Consider the following continuous-time MJLS:

$$
\left\{\begin{aligned}
d x_{t}= & A\left(\phi_{t}\right) x_{t} d t+B\left(\phi_{t}\right) u_{t} d t+E\left(\phi_{t}\right) w_{t} d t \\
& +\sum_{l=1}^{v} \mathbb{W}_{l}\left(\phi_{t}\right) x_{t} d \varpi_{l t} \\
y_{t}= & C_{2}\left(\phi_{t}\right) x_{t}+D_{2}\left(\phi_{t}\right) w_{t} \\
z_{t}= & C_{1}\left(\phi_{t}\right) x_{t}+D_{1}\left(\phi_{t}\right) u_{t}
\end{aligned}\right.
$$

$\left\{\phi_{t}, t \geq 0\right\}$ is a continuous-time homogeneous Markov process with finite state-space $\Xi=\{1, \ldots, \sigma\}$ and transition rate matrix $\Lambda=\left[\lambda_{i j}, i \in \Xi, j \in \Xi\right]$. The set $\Xi$ comprises the operation modes of system (15) and for each possible value of $\theta_{t}=i, i \in \Xi$, we denote the matrices associated with the " $i$-th mode" by: $\mathcal{M}_{i}=\mathcal{M}\left(\theta_{t}=i\right)$. Most of the developed results in the field of MJLS control are obtained under the restrictive assumption of control with complete state observation (Markov and system states). However, the access to the system mode and system state vector may not be possible in some circumstances, which limits the use of such controllers. The design of mode-independent output feedback controller is of great interest in this case. The mode-independent controller used in the rest of the paper is assumed to be of the form: $u_{t}=\mathcal{K} y_{t}$.

We will design the mode-independent static output feedback controller using mode-dependent Lyapunov function approach. This leads to less conservative results when using mode-independent Lyapunov functions.

Proposition 4. System (15) is internally exponentially stabilisable in the mean square sense by static outputfeedback if and only if there exists matrices $\mathcal{P}_{i}=\mathcal{P}_{i}^{\prime}>0$, $\mathbb{X} \in \mathbb{S}^{q}, \mathbb{Y} \in \mathbb{R}^{q \times r}$ and $\mathbb{Z}=\mathbb{Z}^{\prime}>0$ that simultaneously satisfy the following LMI constraints

$$
\begin{aligned}
{\left[\begin{array}{cc}
\mathbb{I} & \mathbf{0} \\
A_{i} & B_{i}
\end{array}\right]^{\prime}\left[\begin{array}{cc}
\Theta_{i} & \mathcal{P}_{i} \\
\mathcal{P}_{i} & \mathbf{0}
\end{array}\right] } & {\left[\begin{array}{cc}
\mathbb{I} & \mathbf{0} \\
A_{i} & B_{i}
\end{array}\right] } \\
& {\left[\begin{array}{cc}
C_{2 i} & \mathbf{0} \\
\mathbf{0} & \mathbb{I}
\end{array}\right]^{\prime}\left[\begin{array}{ll}
\mathbb{X} & \mathbb{Y} \\
\star & \mathbb{Z}
\end{array}\right]\left[\begin{array}{cc}
C_{2 i} & \mathbf{0} \\
\mathbf{0} & \mathbb{I}
\end{array}\right] }
\end{aligned}
$$

and the nonlinear inequality constraint

$$
\mathbb{X} \leq \mathbb{Y}^{-1} \mathbb{Y}^{\prime}
$$

$\forall i \in \Xi$, where $\Theta_{i}=\sum_{l=1}^{v} \mathbb{W}_{l i}^{\prime} \mathcal{P}_{i} \mathbb{W}_{l i}+\sum_{v \in \Xi} \lambda_{i v} \mathcal{P}_{v}$. Let $\left\{\mathcal{P}_{i}, \mathbb{X}, \mathbb{Y}, \mathbb{Z}\right\}$ be a solution, then the nonempty $\{\mathbb{X}, \mathbb{Y}, \mathbb{Z}\}$ ellipsoid is a set of stabilizing gains.

\section{The Control Problem}

\section{$5.1 \mathcal{H}_{\infty}$ Control}

Let us consider the system (1) with

$$
z_{t}=z_{\infty t}=C_{\infty 1} x_{t}+D_{\infty 1}\left(\eta_{t}\right) u\left(y_{t}, \psi_{t}, t\right)
$$

$z_{\infty t}$ stands for the controlled output related to $\mathcal{H}_{\infty}$ performance.

In this section, we deal with the design of controllers that stochastically stabilize the closed-loop system and guarantee the disturbance rejection, with a certain level $\gamma_{\infty}>0$. Mathematically, we are concerned with the characterization of compensators $\varphi_{s}$ that stochastically stabilize the system (3) and guarantee the following for all $w \in L^{2}[0, \infty)$ :

$$
\left\|z_{\infty}\right\|_{\mathcal{E}_{2}}=\mathcal{E}\left\{\int_{0}^{\infty} z_{\infty t}^{\prime} z_{\infty t} d t\right\}^{1 / 2}<\gamma_{\infty}\|w\|_{2}
$$


where $\gamma_{\infty}>0$ is a prescribed level of disturbance attenuation to be achieved. To this end, we need the auxiliary result given by the following proposition.

Proposition 5. If there exists symmetric positivedefinite matrices $\mathcal{P}_{\infty i j k}, i \in Z, j \in S$ and $k \in R$ such that

$$
\left[\begin{array}{cc}
I_{\infty i j k} & \bar{C}_{1 j k}^{\prime} \bar{D}_{1 i j k}+\mathcal{P}_{\infty i j k} \bar{E}_{i j k} \\
\star & \bar{D}_{1 i j k}^{\prime} \bar{D}_{1 i j k}-\gamma_{\infty}^{2} \mathbb{I}
\end{array}\right]<0
$$

where $\beth_{\infty i j k}$ is obtained from $\beth_{i j k}$ by replacing $\mathcal{P}_{i j k}$ by $\mathcal{P}_{\infty i j k}$, then the system (3) is stochastically stable and satisfies (18).

Proof. It follows the same arguments as for the proof of proposition 5.2 in [1].

Using the previous proposition, the following $\mathcal{H}_{\infty}$ control result can be stated.

Proposition 6. If there exists matrices $\mathcal{P}_{\infty i j k}=$ $\mathcal{P}_{\infty i j k}^{\prime}>0, \mathbb{X}_{k} \in \mathbb{S}^{q}, \mathbb{Y}_{k} \in \mathbb{R}^{q \times r}$ and $\mathbb{Z}_{k}=\mathbb{Z}_{k}^{\prime}>0$ that simultaneously satisfy the following LMI constraints

$$
\begin{aligned}
\mathbb{M}_{1 i j}^{\prime}\left[\begin{array}{cc}
\Theta_{i j k} & \mathcal{P}_{\infty i j k} \\
\mathcal{P}_{\infty i j k} & \mathbf{0}
\end{array}\right] \mathbb{M}_{1 i j}<\mathbb{M}_{2 j}^{\prime}\left[\begin{array}{cc}
-\mathbb{I} & \mathbf{0} \\
\mathbf{0} & \gamma_{\infty}^{2} \mathbb{I}
\end{array}\right] \mathbb{M}_{2 j} \\
+\mathbb{M}_{3 i j}^{\prime}\left[\begin{array}{cc}
\mathbb{X}_{k} & \mathbb{Y}_{k} \\
\star & \mathbb{Z}_{k}
\end{array}\right] \mathbb{M}_{3 i j}
\end{aligned}
$$

and the nonlinear constraints (10), where $\mathbb{M}_{1 i j}=$ $\left[\begin{array}{ccc}\mathbb{I} & \mathbf{0} & \mathbf{0} \\ A_{i} & E_{i j} & B_{j}\end{array}\right], \quad \mathbb{M}_{2 j}=\left[\begin{array}{ccc}C_{\infty 1} & \mathbf{0} & D_{\infty 1 j} \\ \mathbf{0} & \mathbb{I} & \mathbf{0}\end{array}\right], \quad \mathbb{M}_{3 i j}=$ $\left[\begin{array}{ccc}C_{2} & D_{2 i j} & \mathbf{0} \\ \mathbf{0} & \mathbf{0} & \mathbb{I}\end{array}\right]$, then the $\left\{\mathbb{X}_{k}, \mathbb{Y}_{k}, \mathbb{Z}_{k}\right\}$-ellipsoids are sets of stabilizing gains such that (18) is verified.

Proof. The proof of this proposition is based on proposition 5 and uses the same arguments as for the proof of proposition 3.

The mode-independent static output feedback $\mathcal{H}_{\infty}$ control result is summarized by the following proposition.

Proposition 7. If there exists matrices $\mathcal{P}_{\infty i}=\mathcal{P}_{\infty i}^{\prime}>0$, $\mathbb{X} \in \mathbb{S}^{q}, \mathbb{Y} \in \mathbb{R}^{q \times r}$ and $\mathbb{Z}=\mathbb{Z}^{\prime}>0$ that simultaneously satisfy the following LMI constraints

$$
\begin{aligned}
\mathbb{M}_{1 i}^{\prime}\left[\begin{array}{cc}
\Theta_{i} & \mathcal{P}_{\infty i} \\
\mathcal{P}_{\infty i} & \mathbf{0}
\end{array}\right] \mathbb{M}_{1 i}<\mathbb{M}_{2 i}^{\prime}\left[\begin{array}{cc}
-\mathbb{I} & \mathbf{0} \\
\mathbf{0} & \gamma_{\infty}^{2} \mathbb{I}
\end{array}\right] \mathbb{M}_{2 i} \\
+\mathbb{M}_{3 i}^{\prime}\left[\begin{array}{cc}
\mathbb{X} & \mathbb{Y} \\
\star & \mathbb{Z}
\end{array}\right] \mathbb{M}_{3 i}
\end{aligned}
$$

and the nonlinear inequality constraint (17), where $\mathbb{M}_{1 i}=\left[\begin{array}{ccc}\mathbb{I} & \mathbf{0} & \mathbf{0} \\ A_{i} & E_{i} & B_{i}\end{array}\right], \mathbb{M}_{2 i}=\left[\begin{array}{ccc}C_{\infty 1 i} & \mathbf{0} & D_{\infty 1 i} \\ \mathbf{0} & \mathbb{I} & \mathbf{0}\end{array}\right], \mathbb{M}_{3 i}=$ $\left[\begin{array}{ccc}C_{2 i} & D_{2 i} & \mathbf{0} \\ \mathbf{0} & \mathbf{0} & \mathbb{I}\end{array}\right]$, then the $\{\mathbb{X}, \mathbb{Y}, \mathbb{Z}\}$-ellipsoid is a set of stabilizing gains such that (18) is satisfied.

\section{2 $\mathcal{H}_{2}$ Control}

Consider the system (1) with

$$
z_{t}=z_{2 t}=C_{21} x_{t}+D_{21}\left(\eta_{t}\right) u\left(y_{t}, \psi_{t}, t\right)
$$

and $D_{2}\left(\xi_{t}, \eta_{t}\right)=\mathbf{0} . z_{2 t}$ stands for the controlled output related to $\mathcal{H}_{2}$ performance.

Before introducing the $\mathcal{H}_{2}$ control result, let us consider the following definition.

Definition 4. We define the $\mathcal{H}_{2}$-norm of the IESS system $\left(\varphi_{c l}\right)$ as

$$
\left\|\varphi_{c l}\right\|_{2}^{2}=\sum_{d=1}^{m} \sum_{i, j, k} \mu_{i j k}\left\|z_{d, i, j, k}\right\|_{\mathcal{E}_{2}}^{2}
$$

where $z_{d, i, j, k}$ is the controlled output of the system with initial conditions $\xi_{0}=i, \eta_{0}=j, \psi_{0}=k$, disturbed by $w_{t}=e_{d} \delta_{t} . e_{d}$ is a $m$-dimensional unitary vector with its $d$ th entry as 1 and other entries as $0^{\prime}$ s. $\delta_{t}$ is an impulse function and $\mu_{i j k}$ is the initial probability distribution for $\xi_{0}=i, \eta_{0}=j, \psi_{0}=k$.

From the definition above and using the same arguments as in $[6,26,27]$, we can state the following proposition.

Proposition 8. Assume that $\left(\varphi_{c l}\right)$ is IESS then

i) $\left\|\varphi_{c l}\right\|_{2}^{2}=\sum_{i, j, k} \mu_{i j k} \operatorname{tr}\left(E_{i j}^{\prime} \mathcal{P}_{o i j k} E_{i j}\right)$, where $\mathcal{P}_{o}=$ $\left\{\mathcal{P}_{o 111}, \ldots, \mathcal{P}_{\text {oszr }}\right\}$ denotes the observability Grammian, i.e., $\mathcal{P}_{o i j k}$ are the unique positive semi definite solutions of the following equations:

$$
\beth_{o i j k}+\bar{C}_{21}^{\prime} \bar{C}_{21}=0
$$

ii) $\left\|\varphi_{c l}\right\|_{2}^{2}<\sum_{i, j, k} \mu_{i j k} \operatorname{tr}\left(E_{i j}^{\prime} \mathcal{P}_{2 i j k} E_{i j}\right)$, where $\mathcal{P}_{2 i j k}$ is a positive definite solution of the following matrix inequality

$$
\beth_{2 i j k}+\bar{C}_{21}^{\prime} \bar{C}_{21}<0
$$

iii) If there exists positive definite matrices $\mathcal{P}_{2 i j k}$ and matrices $\mathcal{K}_{k}$ such that

$$
\begin{gathered}
\sum_{i, j, k} \mu_{i j k} \operatorname{tr}\left(E_{i j}^{\prime} \mathcal{P}_{2 i j k} E_{i j}\right)<\gamma_{2}^{2} \\
\beth_{2 i j k}+\bar{C}_{21}^{\prime} \bar{C}_{21}<0
\end{gathered}
$$

Then $\mathcal{K}_{k}$ are stabilizing gains such that $\left\|\varphi_{c l}\right\|_{2}<\gamma_{2}$.

Using the same framework as for the stochastic stabilization and the $\mathcal{H}_{\infty}$ control problematic, similar $\mathcal{H}_{2}$ control results are obtained and summarized as follows:

Proposition 9. If there exists matrices $\mathcal{P}_{2 i j k}=\mathcal{P}_{2 i j k}^{\prime}>$ $0, \mathbb{X}_{k} \in \mathbb{S}^{q}, \mathbb{Y}_{k} \in \mathbb{R}^{q \times r}$ and $\mathbb{Z}_{k}=\mathbb{Z}_{k}^{\prime}>0$ that simultaneously satisfy the constraints (10), (24) and

$$
\mathbb{N}_{1 i j}^{\prime}\left[\begin{array}{cc}
\Theta_{i j k} & \mathcal{P}_{2 i j k} \\
\mathcal{P}_{2 i j k} & \mathbf{0}
\end{array}\right] \mathbb{N}_{1 i j}<-\mathbb{N}_{2 j}^{\prime} \mathbb{N}_{2 j}+\mathbb{N}_{3}^{\prime}\left[\begin{array}{cc}
\mathbb{X}_{k} & \mathbb{Y}_{k} \\
\star & \mathbb{Z}_{k}
\end{array}\right] \mathbb{N}_{3}
$$

$\mathbb{N}_{1 i j}=\left[\begin{array}{cc}\mathbb{I} & \mathbf{0} \\ A_{i} & B_{j}\end{array}\right], \quad \mathbb{N}_{2 j}=\left[\begin{array}{ll}C_{21} & D_{21 j}\end{array}\right], \quad \mathbb{N}_{3}=$ $\left[\begin{array}{cc}C_{2} & \mathbf{0} \\ \mathbf{0} & \mathbb{I}\end{array}\right]$, then the $\left\{\mathbb{X}_{k}, \mathbb{Y}_{k}, \mathbb{Z}_{k}\right\}$-ellipsoids are sets of 
stabilizing gains such that $\left\|\varphi_{c l}\right\|_{2}<\gamma_{2}$.

We conclude this section by giving, as in the previous sections, results relating to mode-independent $\mathcal{H}_{2}$ control of MJLS.

Proposition 10. If there exist matrices $\mathcal{P}_{2 i}=\mathcal{P}_{2 i}^{\prime}>0$, $\mathbb{X} \in \mathbb{S}^{q}, \mathbb{Y} \in \mathbb{R}^{q \times r}$ and $\mathbb{Z}=\mathbb{Z}^{\prime}>0$ that simultaneously satisfy the constraints $(17), \sum_{i} \mu_{i} \operatorname{tr}\left(E_{i}^{\prime} \mathcal{P}_{2 i} E_{i}\right)<\gamma_{2}^{2}$ and

$$
\begin{aligned}
& \mathbb{N}_{1 i}^{\prime}\left[\begin{array}{cc}
\Theta_{i} & \mathcal{P}_{2 i} \\
\mathcal{P}_{2 i} & \mathbf{0}
\end{array}\right] \mathbb{N}_{1 i}<-\mathbb{N}_{2 i}^{\prime} \mathbb{N}_{2 i}+\mathbb{N}_{3 i}^{\prime}\left[\begin{array}{cc}
\mathbb{X} \mathbb{Y} \\
\star \mathbb{Z}
\end{array}\right] \mathbb{N}_{3 i} \quad(26) \\
& \mathbb{N}_{1 i}=\left[\begin{array}{cc}
\mathbb{I} & \mathbf{0} \\
A_{i} & B_{i}
\end{array}\right], \quad \mathbb{N}_{2 i}=\left[\begin{array}{ll}
C_{21 i} & D_{21 i}
\end{array}\right], \mathbb{N}_{3 i}= \\
& {\left[\begin{array}{cc}
C_{2 i} & \mathbf{0} \\
\mathbf{0} & \mathbb{I}
\end{array}\right], \text { then the }\{\mathbb{X}, \mathbb{Y}, \mathbb{Z}\} \text {-ellipsoid is a set of stabi- }}
\end{aligned}
$$
lizing gains such that $\left\|\varphi_{c l}\right\|_{2}<\gamma_{2}$.

\section{$5.3 \mathcal{H}_{2} / \mathcal{H}_{\infty}$ Synthesis}

The $\mathcal{H}_{2} / \mathcal{H}_{\infty}$ synthesis problem consists in finding a common controller that stochastically stabilizes the system and ensures the different performance levels. This can be stated as follows:

For two given levels on the $\mathcal{H}_{\infty}$ and $\mathcal{H}_{2}$ norms, $\gamma_{\infty}$ and $\gamma_{2}$ respectively, find stabilizing static output feedback gains $\mathcal{K}_{k}$ such that

$$
\begin{gathered}
\left\|z_{\infty}\right\|_{\mathcal{E}_{2}}<\gamma_{\infty}\|w\|_{2} \\
\left\|\varphi_{c l}\right\|_{2}<\gamma_{2}
\end{gathered}
$$

Corollary 1. If there exists matrices $\mathcal{P}_{\infty i j k}, \mathcal{P}_{2 i j k}, \mathbb{X}_{k} \in$ $\mathbb{S}^{q}, \mathbb{Y}_{k} \in \mathbb{R}^{q \times r}$ and $\mathbb{Z}_{k} \in \mathbb{S}^{r}$ that simultaneously satisfy the constraints (10), (20) and (24)-(25), then the $\left\{\mathbb{X}_{k}, \mathbb{Y}_{k}, \mathbb{Z}_{k}\right\}$-ellipsoids are sets of stabilizing gains such that the performances levels are satisfied.

\section{Computational Issues and Examples}

\subsection{A Cone Complementary Algorithm (CCL)}

In this section, an iterative algorithm is proposed to solve the different synthesis conditions. It is based on a cone complementary technique [12]. The proposed CCL algorithm is based on the following lemma which is obtained using the same arguments as in [20].

Lemma 1. The $\mathcal{H}_{2} / \mathcal{H}_{\infty}$ control problem is feasible if and only if zero is the global optimum of the optimisation problem

$$
\left\{\begin{array}{l}
\min \quad \operatorname{tr}(\mathbb{T S}) \\
\text { s.t. } \quad(20),(24),(25) \\
\mathbb{X}_{k} \leq \widehat{\mathbb{X}}_{k} \quad \mathcal{S}_{k}=\left[\begin{array}{cc}
\widehat{\mathbb{X}}_{k} & \mathbb{Y}_{k} \\
\star & \mathbb{Z}_{k}
\end{array}\right] \geq 0 \\
\mathcal{T}_{1 k} \geq \mathbb{I} \quad \mathcal{T}_{k}=\left[\begin{array}{cc}
\mathcal{T}_{1 k} & \mathcal{T}_{2 k} \\
\star & \mathcal{T}_{3 k}
\end{array}\right] \geq 0
\end{array}\right.
$$

where $\mathbb{S}=\operatorname{diag}\left\{\mathcal{S}_{1}, \ldots, \mathcal{S}_{r}\right\}, \quad \mathbb{T}=\operatorname{diag}\left\{\mathcal{T}_{1}, \ldots, \mathcal{T}_{r}\right\}$. CCL algorithm. For two given levels $\gamma_{\infty}, \gamma_{2}$

i) Find a feasible solution $\mathbb{X}_{k 0}, \mathbb{Y}_{k 0}, \mathbb{Z}_{k 0}, \hat{\mathbb{X}}_{k 0}, \mathcal{P}_{2 i j k 0}$, $\mathcal{P}_{\infty i j k 0}, \mathbb{T}_{0}, \mathbb{S}_{0}$. If there is no solution, STOP, the algorithm failed. $h=0$;

ii) set $\mathbf{V}_{h}=\mathbb{S}_{h}, \mathbf{W}_{h}=\mathbb{T}_{h}$, and find $\mathbb{X}_{k(h+1)}, \mathbb{Y}_{k(h+1)}$, $\mathbb{Z}_{k(h+1)}, \hat{\mathbb{X}}_{k(h+1)}, \mathcal{P}_{2 i j k(h+1)}, \mathcal{P}_{\infty i j k(h+1)}, \mathbb{T}_{h+1}, \mathbb{S}_{h+1}$, solutions of the LMI problem

$$
\left\{\begin{array}{l}
\min \quad \operatorname{tr}\left(\mathbf{V}_{h} \mathbb{T}+\mathbf{W}_{h} \mathbb{S}\right) \\
\text { s.t. } \quad(20),(24),(25) \\
\mathbb{X}_{k} \leq \widehat{\mathbb{X}}_{k} \quad \mathcal{S}_{k}=\left[\begin{array}{cc}
\widehat{\mathbb{X}}_{k} & \mathbb{Y}_{k} \\
\star & \mathbb{Z}_{k}
\end{array}\right] \geq 0 \\
\mathcal{T}_{1 k} \geq \mathbb{I} \quad \mathcal{T}_{k}=\left[\begin{array}{cc}
\mathcal{T}_{1 k} & \mathcal{T}_{2 k} \\
\star & \mathcal{T}_{3 k}
\end{array}\right] \geq 0
\end{array}\right.
$$

iii) if $\operatorname{tr}\left(\mathcal{T}_{h-1} \mathcal{S}_{h-1}-\mathcal{T}_{h} \mathcal{S}_{h}<\varepsilon\right)$, then STOP, the algorithm failed (slow progress).

iv) if $\mathbb{X}_{k} \leq \mathbb{Y}_{k} \mathbb{Z}_{k}^{-1} \mathbb{Y}_{k}^{\prime}$, STOP, matrix ellipsoids are found. Otherwise, set $h=h+1$ and go to step ii).

\subsection{Numerical Example}

We applied the proposed mode-independent output feedback $\mathcal{H}_{2} / \mathcal{H}_{\infty}$ control to a VTOL helicopter model adapted from [8]. The dynamics can be written as:

$$
\left\{\begin{array}{l}
d x_{t}=A\left(\phi_{t}\right) x_{t} d t+B\left(\phi_{t}\right) u_{t} d t+E w_{t} d t+\mathbb{W}_{1} x_{t} d \varpi_{t} \\
y_{t}=C_{2} x_{t} \\
z_{\infty t}=C_{\infty 1} x_{t}+D_{\infty 1} u_{t} \\
z_{2 t}=C_{21} x_{t}+D_{21} u_{t}
\end{array}\right.
$$

where $\phi_{t}$ indicates the airspeed. The different parameters are given in [8]. The behavior of $\phi_{t}$ is modelled as a Markov chain with three different states, corresponding to airspeeds of 135 (nominal value), 60, 170 Knots. The transition matrix is given by:

$$
\Xi=\left[\begin{array}{ccc}
-0.0907 & 0.0671 & 0.0236 \\
0.0671 & -0.0671 & 0 \\
0.0236 & 0 & -0.0236
\end{array}\right]
$$

The central controller corresponding to $\gamma_{\infty}=\gamma_{2}=1$ (center of the ellipsoid) is given by

$$
\mathcal{K}_{0}^{\prime}=[0.5143-0.4358]
$$

The state trajectories of the closed loop system resulting from the obtained controller are shown in Figure 1. Figure 2 represents the evolution of the controlled outputs $z_{\infty t}$. It can be seen that the closed-loop system is stochastically stable and that the disturbance attenuation is achieved. 


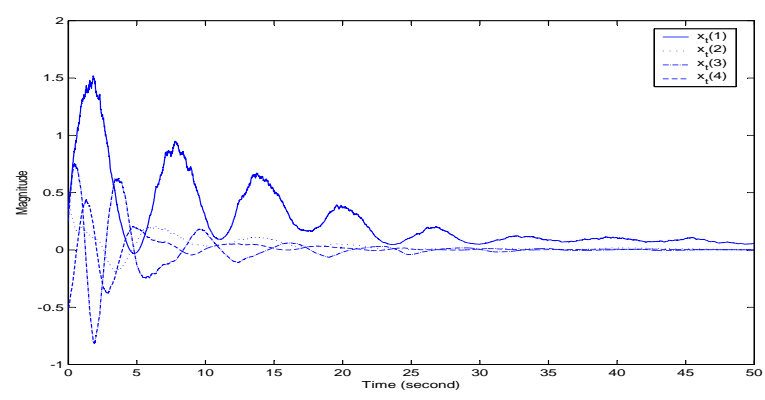

Fig. 1. States of the closed loop system: single sample path simulation

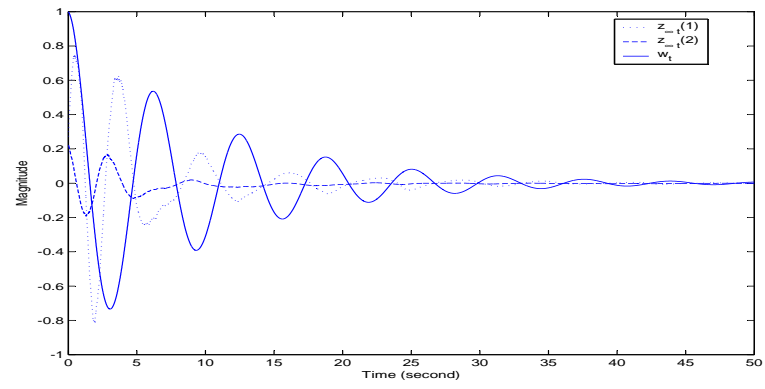

Fig. 2. Evolution of the controlled outputs $z_{\infty t}$ : single sample path simulation

\section{Conclusion}

In this paper, the static output feedback $\mathcal{H}_{2} / \mathcal{H}_{\infty}$ control of continuous time reconfigurable systems with Markovian processes and state-dependent noise was considered. We have proposed a synthesis method of reconfigurable controllers that take explicitly into account the inherent imperfections of the FDI process. Another problematic related to MJLS control was also addressed. This problematic concerns the mode-independent static output feedback $\mathcal{H}_{2} / \mathcal{H}_{\infty}$ control of MJLS with multiplicative noise. The numerical resolution of the obtained results was done using a cone complementary algorithm and its running was illustrated on a numerical example.

\section{References}

[1] S. Aberkane, J.C. Ponsart, and D. Sauter. Output feedback $\mathcal{H}_{\infty}$ control of a class of stochastic hybrid systems with wiener process via convex analysis. Int. J. Innovative Computing, Information and Control, 2:123-126, 2006.

[2] E. K. Boukas. Exponential stabilizability of stochastic systems with markovian jumping parameters. Automatica, 35:1437-1441, 1999

[3] E. K. Boukas. Stochastic Switching Systems: Analysis and Design. Birkhauser, Boston, 2001.

[4] E. K. Boukas. Static output feedback control for stochastic hybrid systems: Lmi approach. Automatica, 42:183-188, 2006.

[5] E. K. Boukas and N. F. Al-Muthairi. $\mathcal{H}_{\infty}$ constant gain state feedback of stochastic hybrid systems with wienner process. Mathematical Problems in Engineering, 4:333-345, 2004.
[6] O. L. V. Costa, J. B. R. do Val, and J. C. Geromel. Continuous-time state-feedback $\mathcal{H}_{2}$-control of Markovian jump linear systems via convex analysis. Automatica, 35:259268, 1999.

[7] O. L. V. Costa, E. O. A. Filho, E. K. Boukas, and R. P. Marques. Constrained quadratic state feedback control of discrete-time markovian jump linear systems. Automatica, 35:617-626, 1999.

[8] D. P. de Farias, J. C. Geromel, J. B. R. do Val, and O. L. V. Costa. Output feedback control of Markov jump linear systems in continuous-time. IEEE Transactions on Automatic Control, 45:944-949, 2000.

[9] C. E. de Souza and M. D. Fragoso. $\mathcal{H}_{\infty}$ control for linear systems with Markovian jumping parameters. Control Theory and Advanced Technology, 9:457-466, 1993.

[10] J. B. R. do Val, J. C. Geromel, and A. P. C. Gonçalves. The $\mathcal{H}_{2}$ control for jump linear systems: cluster observations of the Markov state. Automatica, 38:343-349, 2002.

[11] L. El-Ghaoui and M. Ait-Rami. Robust state-space stabilization of jump linear systems via lmis. International Journal of Robust and Nonlinear Control, 6:1015-1022, 1996.

[12] L. El Ghaoui, F. Oustry, and M. AitRami. A cone complementary linearization algorithm for static outputfeedback and related problems. IEEE Transactions on Automatic Control, 42:1171-1176, 1997.

[13] Y. Ji and H. J. Chizeck. Controllability, stabilizability, and continuous-time Markovian jump linear quadratic control. IEEE Transactions on Automatic Control, 35:777-788, 1990.

[14] Y. Ji and H. J. Chizeck. Jump linear quadratic Gaussian control in continuous time. IEEE Transactions on Automatic Control, 37:1884-1892, 1992.

[15] H. J. Kushner. Stochastic Stability and Control. Academic Press, New York, 1967.

[16] M. Mahmoud, J. Jiang, and Y. Zhang. Stochastic stability analysis of active fault-tolerant control systems in the presence of noise. IEEE Transactions on Automatic Control, 46:1810-1815, 2001

[17] M. Mahmoud, J. Jiang, and Y. Zhang. Active Fault Tolerant Control Systems: Stochastic Analysis and Synthesis. Springer, 2003.

[18] M. Mariton. Detection delays, false alrm rates and the reconfiguration of control systems. International Journal of Control, 42:459-465, 1989.

[19] M. Mariton. Jump Linear Systems in Automatic Control. Marcel Dekker, New York, 1990.

[20] D. Peaucelle and D. Arzelier. Ellipsoidal sets for resilient and robust static output-feedback. IEEE Transaction on Automatic Control, 50:899-904, 2005.

[21] P. Shi and E. K. Boukas. $\mathcal{H}_{\infty}$-control for Markovian jumping linear systems with parametric uncertainty. Journal of Optimization Theory and Applications, 95:75-99, 1997.

[22] P. Shi, E. K. Boukas, S. K. Nguang, and X. Guo. Robust disturbance attenuation for discrete-time active fault tolerant control systems with uncertainties. Optimal Control Applications and Methods, 24:85-101, 2003.

[23] R. E. Skelton, T. Iwasaki, and K. Grigoriadis. A Unified Algebraic Approach to Linear Control Design. Taylor and Francis, 1998.

[24] R. Srichander and B. K. Walker. Stochastic stability analysis for continuous-time fault tolerant control systems. International Journal of Control, 57:433-452, 1993. 
[25] V. L. Syrmos, C. T. Abdallah, P. Dorato, and K. Grigoriadis. Static output feedback: A survey. Automatica, 33:125-137, 1997.

[26] T. Morozan V. Dragan and A. Stoica. $\mathcal{H}_{2}$ optimal control for linear stochastic systems. Automatica, 40:1103-1113, 2004.

[27] T. Morozan V. Dragan and A. Stoica. Mathematical Methods in Robust Control of Linear Stochastic Systems. Springer, 2006. 